Artículo

\title{
Caracterización de agroecosistemas de maíz en la planicie costera del Istmo, Oaxaca
}

\author{
Ernesto Castañeda-Hidalgo ${ }^{2 \S}$ \\ Víctor Ernesto Rasgado-Cabrera ${ }^{1}$ \\ Gisela Margarita Santiago-Martínez ${ }^{2}$ \\ Salvador Lozano-Trejo ${ }^{2}$ \\ María Isabel Pérez-León ${ }^{2}$ \\ Yuri Villegas-Aparicio ${ }^{2}$ \\ ${ }^{1}$ Maestría en Ciencias en Productividad en Agroecosistemas-Instituto Tecnológico del Valle de Oaxaca. \\ (agrovic.vr@gmail.com). ${ }^{2}$ Instituto Tecnológico del Valle de Oaxaca-Tecnológico Nacional de México. \\ (gissant68@hotmail.com; lozanos2004@gmail.com; leonisa70@hotmail.com; yuriva1968@gmail.com). \\ ${ }^{\S}$ Autor para correspondencia: casta_h50@ hotmail.com.
}

\section{Resumen}

Los agroecosistemas son ecosistemas transformados por el humano al usar los recursos para producir alimentos, fibras y materias primas. El estudio se realizó en 2017 con el objetivo de identificar y caracterizar los agroecosistemas de maíz en la planicie costera del Istmo Oaxaca y conocer su estado actual. Se utilizaron las metodologías del sondeo rural participativo y la de estudio de agroecosistemas. Se aplicó un cuestionario a una muestra de 60 productores de maíz. La información se sistematizó en Microsoft Excel 2010 y procesó en SAS ${ }^{\circledR}$ V9.0 para el análisis de componentes principales (ACP) y discriminación canónica. De 103 variables, se seleccionaron 10 por ACP y 12 incorporadas a juicio de valor, en las dimensiones biofísica, social, económica y tecnológica. Se identificaron tres agroecosistemas (distancia cuadrada de Mahalanobis y pruebas multivariables con aproximación de F) ( $p<0.01)$ : 1) agroecosistema de maíz Juchitán de ZaragozaChicapa de Castro (AE1); 2) agroecosistema de maíz Álvaro Obregón-Emiliano Zapata (AE2); y 3) agroecosistema de maíz La Venta-La Ventosa (AE3). En los tres AE siembran maíz Zapalote Chico de forma tradicional y baja mecanización (preparación de suelos y siembra). Aunque los rendimientos son muy similares en los tres AE, en ingreso mensual sobresalen los AE1 y AE3, en contraste con el AE2, donde es menor, debido a su actividad ganadera (cabras en pastoreo intensivo) distinta a los otros AE (ganado de doble propósito), baja superficie de cultivo, pocos jornales contratados, etc. El maíz es para autoconsumo, aunque se venden excedentes en el mercado local.

Palabras clave: Zea mays L., agricultura tradicional, Istmo de Tehuantepec, rendimiento anual.

Recibido: abril de 2020

Aceptado: agosto de 2020 


\section{Introducción}

El maíz (Zea mays L.) es uno de los tres cereales más importantes en el mundo. Es fuente para la alimentación humana y para la ganadería (González et al., 2013). En México, es la especie cultivada más importante y diversa y supera al sorgo, trigo, cebada, arroz y avena (López et al., 2010; SAGARPA, 2017).

La alta diversidad de maíces en México se manifiesta en los sistemas agrícolas tradicionales, donde utilizan variedades autóctonas adaptadas a las condiciones locales. Son preferidos y componente clave en la seguridad alimentaria del sector rural (Cabrera et al., 2018). Se siembran 9.6 millones de hectáreas anuales con un rendimiento nacional promedio de $2.2 \mathrm{t} \mathrm{ha}^{-1}$.

El consumo nacional per capita en 2015 fue de $297 \mathrm{~kg}$ y en el sur de Oaxaca, superior a los $350 \mathrm{~kg}$ (SIAP, 2016). En el 2016, Oaxaca fue sexto lugar con 552438.33 ha y un rendimiento promedio de $1.31 \mathrm{t} \mathrm{ha}^{-1}$ (SIAP, 2017). El 90\% de los agricultores cultivan maíz en diferentes altitudes ( 0 a 2 $800 \mathrm{~m}$ ) y diferentes condiciones edafoclimáticas; lo que ha permitido identificar más de treinta razas ampliamente utilizadas en la alimentación (Aragón et al., 2005; Salinas et al., 2013).

La planicie costera del Istmo de Tehuantepec es una importante zona agrícola (López-Romero et al., 2005) y el cultivo de maíz de la raza Zapalote Chico es el más representativo, 73\% de las 111 000 ha cultivadas (López et al., 2009; López et al., 2010). El 85\% es de temporal con rendimientos promedio de $1.3 \mathrm{t} \mathrm{ha}^{-1}$ (SIAP, 2015). Este genotipo es muy precoz y permite tres cosechas al año. Su bajo porte evita el efecto de los fuertes vientos y se elabora el Totopo (tortilla de maíz martajado con textura crujiente y con perforaciones al interior) y otros alimentos de la población de descendencia zapoteca (CONABIO, 2010; INEGI, 2014; Cabrera et al., 2018).

Los agroecosistemas (AE) son ecosistemas transformados y proporcionan recursos naturales para actividades agropecuarias. Cada AE posee características propias que los diferencian de otros (Hernández-Xolocotzi y Ramos, 1977; Masera et al., 2000). Los AE tradicionales tienen una connotación socioeconómica y cultural ligada a la valoración, apropiación y usufructo de recursos locales por el humano y la sociedad. Las características de los AE están determinadas por factores biofísicos, socioculturales, económicos, tecnológicos, finalidad de la producción, entre otros (Masera et al., 2000).

Estos factores son igualmente utilizados en el proceso de caracterización de los AE, que tiene como objetivo, agrupar sistemas agrícolas que operen de manera similar para conocer su situación y problemática actual o para estudiar su evolución en el tiempo (Vilaboa-Arroniz et al., 2009).

Los estudios relacionados con los AE de maíz en la planicie costera del Istmo Oaxaca se centran en aspectos técnico-productivos, pero no contemplan aspectos sociales, culturales y de organización del campesinado en el proceso productivo. Por ello, el presente estudio se desarrolló con el objetivo de identificar y caracterizar a los agroecosistemas de maíz en la zona y conocer su estado actual. 


\section{Materiales y métodos}

\section{Características del área de estudio}

El estudio se realizó en Juchitán de Zaragoza, que posee la mayor superficie de la planicie costera del Istmo de Tehuantepec, Oaxaca y es coincidente con el Distrito de Riego No. 19. Comprende, además de la cabecera municipal, cinco agencias municipales y dos agencias de policía.

Se localiza entre los paralelos $16^{\circ} 12^{\prime}$ y $16^{\circ} 38^{\prime}$ de latitud norte y $94^{\circ} 44^{\prime}$ y $95^{\circ} 08^{\prime}$ longitud oeste, en un rango altitudinal de 0 a $500 \mathrm{~m}$. El clima es un $\mathrm{Aw}_{0}$ (w) ig, catalogado como cálido subhúmedo, el más seco de los subhúmedos, con lluvias en verano. Temperatura media de $26^{\circ} \mathrm{C}$ y una precipitación media anual de $978 \mathrm{~mm}$. La vegetación natural se extiende sobre una gran planicie e incluye selvas bajas caducifolias y matorrales espinosos. Predominan los suelos Vertisoles, Phaeozem, Arenosoles, Luvisoles, Fluvisoles, Cambisoles, Solonchak y Gleysoles (INEGI, 2010a; 2010b; UABJO, 2014).

\section{Proceso metodológico}

La investigación se fundamentó en la investigación-acción participativa acorde a Colmenares (2012). El enfoque fue mixto, del tipo exploratorio y descriptivo. El diseño fue no-experimental y los datos se generaron directamente sin procesamiento previo. El criterio para la selección de las comunidades fue la dedicación a la actividad agrícola, al existir comunidades totalmente pesqueras.

La investigación contempló seis comunidades del municipio: Juchitán de Zaragoza, Álvaro Obregón, Emiliano Zapata, La Ventosa, La Venta y Chicapa de Castro. La metodología diagnóstica para analizar a las comunidades y su contexto fue el sondeo rural participativo (SRP) de Selener et al. (1999). Para generar la información de campo se utilizaron la encuesta mediante la aplicación de un cuestionario, el diálogo con los productores y los recorridos de campo.

El cuestionario se estructuró en dos apartados, el diagnóstico para identificar las características sociales, económicas, productivas y de organización en las unidades de producción campesinas, a partir de su análisis se llegó al diagnóstico. El segundo fue para la caracterización de los sistemas de cultivo, mediante la metodología para el estudio de agroecosistemas de Hernández-Xolocotzi (1977). Se aplicó el cuestionario a agricultores por estrato (localidades). El criterio de selección fue que fueran maiceros y pertenecieran a los 1200 registrados en el padrón del CADER.

El tamaño de la muestra fue de 60 productores (5\% de la población); 22, 20 y 18 para los AE1, AE2 y AE3, respectivamente. La selección se realizó por la técnica de la 'bola de nieve'. Consiste en un primer acercamiento con un conocedor local a quien se le aplica el cuestionario; posteriormente nombra a los posibles informantes clave en la comunidad.

Los datos generados se clasificaron en los ejes ambiental, económico, social y tecnológico para su análisis. Se propusieron de inicio 103 variables de estudio, para reducir la dimensionalidad se aplicó un análisis de componentes principales (ACP) (SAS). Con este análisis se seleccionaron 10 variables de mayor peso relativo, cinco del eje biofísico, dos del social y tres del económico. 
En el eje tecnológico no hubo variables debido a su homogeneidad. El segundo criterio para seleccionar las variables fue el 'juicio de valor' acorde a Masera et al. (2000), para incorporar variables para la caracterización de los AE: eje social (2); económico (2); y tecnológico (8). Finalmente se consideraron 22 variables en los ejes biofísico (5); sociales (4); económicas (5); y tecnológicas (8) (Cuadro 1).

Cuadro 1. Variables determinantes para la caracterización de los agroecosistemas.

\begin{tabular}{|c|c|c|c|}
\hline \multirow[b]{2}{*}{ Eje } & \multicolumn{2}{|r|}{ Variables } & \multirow[b]{2}{*}{ Total } \\
\hline & $\begin{array}{c}\text { Análisis } \\
\text { multivariado }\end{array}$ & Juicio de valor & \\
\hline$\frac{8}{\sqrt[0]{\infty}}$ & $\begin{array}{l}\text { 1. T. media } \\
\text { máxima (mmax) } \\
\text { 2. T. media } \\
\text { mínima (mmin) } \\
\text { 3. T. media anual } \\
\text { (ma) } \\
\text { 4. Precipitación } \\
\text { (pp) } \\
\text { 5. Altitud } \\
\text { (msnm) }\end{array}$ & & 5 \\
\hline 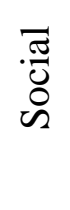 & $\begin{array}{l}\text { 1. Etnia (etn) } \\
\text { 2. Integrantes de } \\
\text { la familia (itfam) }\end{array}$ & $\begin{array}{l}\text { 1. Integrantes de la familia que participan en la unidad de } \\
\text { producción (ifup) } \\
\text { 2. Organización (org) }\end{array}$ & 4 \\
\hline 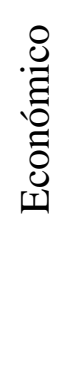 & $\begin{array}{l}\text { 1. Ingreso } \\
\text { mensual (imens) } \\
\text { 2. Acceso a } \\
\text { PROAGRO } \\
\text { (proagr) } \\
\text { 3. Monto de } \\
\text { mano de obra } \\
\text { (mmdeo) }\end{array}$ & $\begin{array}{l}\text { 1. Fuentes de ingreso (fuening) } \\
\text { 2. Precio de maíz (pmaíz) }\end{array}$ & 5 \\
\hline $\begin{array}{l}0 \\
\frac{8}{00} \\
\frac{0}{0} \\
\stackrel{0}{0} \\
\Theta\end{array}$ & & $\begin{array}{l}\text { 1. Labranza (lab); 2. Siembra (siemb); 3. Prácticas de } \\
\text { conservación de suelo (cons); 4. Insumos (insum); } 5 \text {. } \\
\text { Superficie de siembra (sups); 6. Número de parcelas (npar); } \\
\text { 7. Asesoría técnica (atecni); y 8. Rendimiento acumulado (kg } \\
\text { ha }^{-1} \text { ) (racum) }\end{array}$ & 8 \\
\hline
\end{tabular}

Se realizó un análisis de discriminantes canónicos (ADC) con el procedimiento CANDISC (SAS) para detectar discrepancias entre localidades. Se utilizó la distancia cuadrada de Mahalanobis y pruebas multivariables con aproximación de F. 


\section{Resultados y discusión}

\section{Identificación y caracterización de agroecosistemas}

De acuerdo con el estudio de las variables determinantes, en las funciones discriminantes de máxima separación ( $p<0.01$ ), se identificaron tres agroecosistemas: 1) agroecosistema de maíz en Juchitán de Zaragoza-Chicapa de Castro; 2) agroecosistema de maíz en Álvaro Obregón-Emiliano Zapata; y 3) agroecosistema de maíz La Venta-La Ventosa (Figura 1).

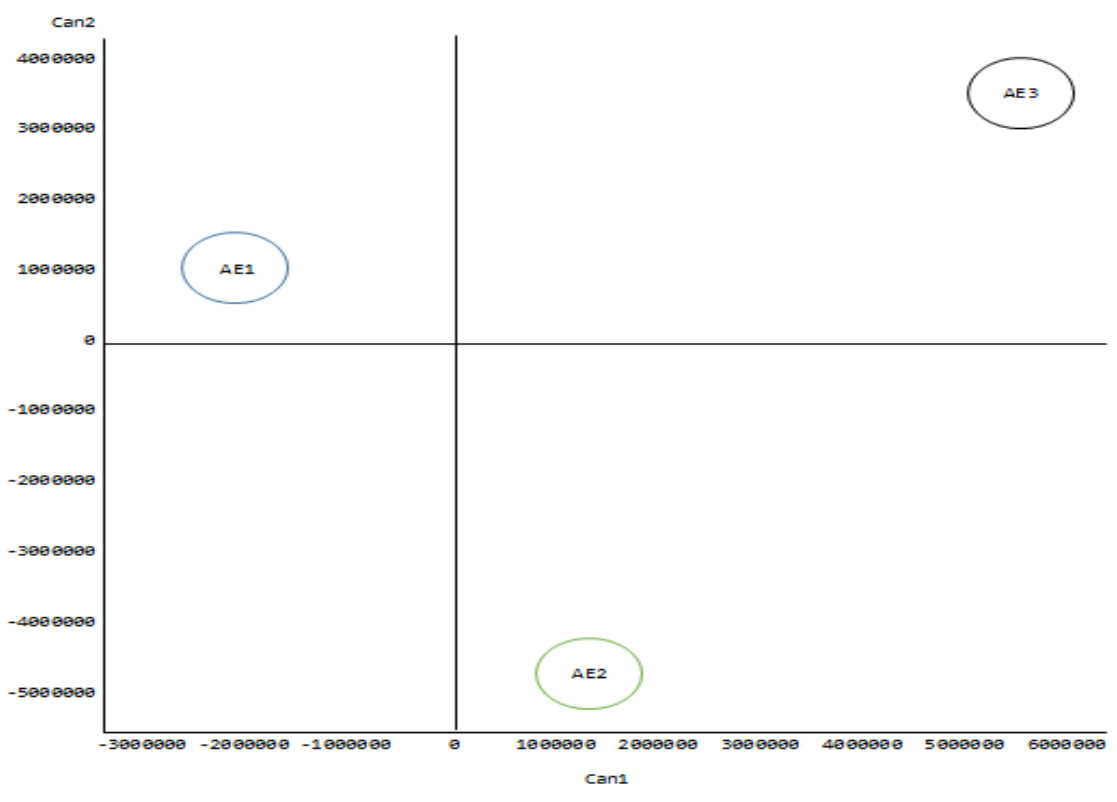

Figura 1. Separación de agroecosistemas por discriminación canónica con sus variables determinantes.

\section{Agroecosistema de maíz en Juchitán de Zaragoza-Chicapa de Castro (AE1)}

El AE1 se ubica en las localidades de Juchitán de Zaragoza y Chicapa de Castro, en altitudes de hasta $22 \mathrm{~m}$; temperatura media anual de $27{ }^{\circ} \mathrm{C}$ y máximas y mínimas de 31.7 y $16.8{ }^{\circ} \mathrm{C}$, respectivamente. La precipitación promedio anual es de $980 \mathrm{~mm}$. Aragón et al. (2005) indican que en Oaxaca, el maíz se cultiva de 0 a 2800 msnm bajo diferentes condiciones climáticas y edáficas.

De acuerdo con Cervantes-Herrera et al. (2015), el maíz produce aceptablemente en zonas donde llueve anualmente de 600 a $1000 \mathrm{~mm}$ bien distribuidos durante cuatro a siete meses. Comprende a campesinos zapotecas (100\%) con unidades de producción campesina (UPC) integradas por cinco individuos, el jefe de familia con la colaboración de dos hijos. Esto es ventajoso, Serrano-Ojeda et al. (2016), reportan que en el centro de Puebla las UPF se integran por cuatro personas (Figura 2). 


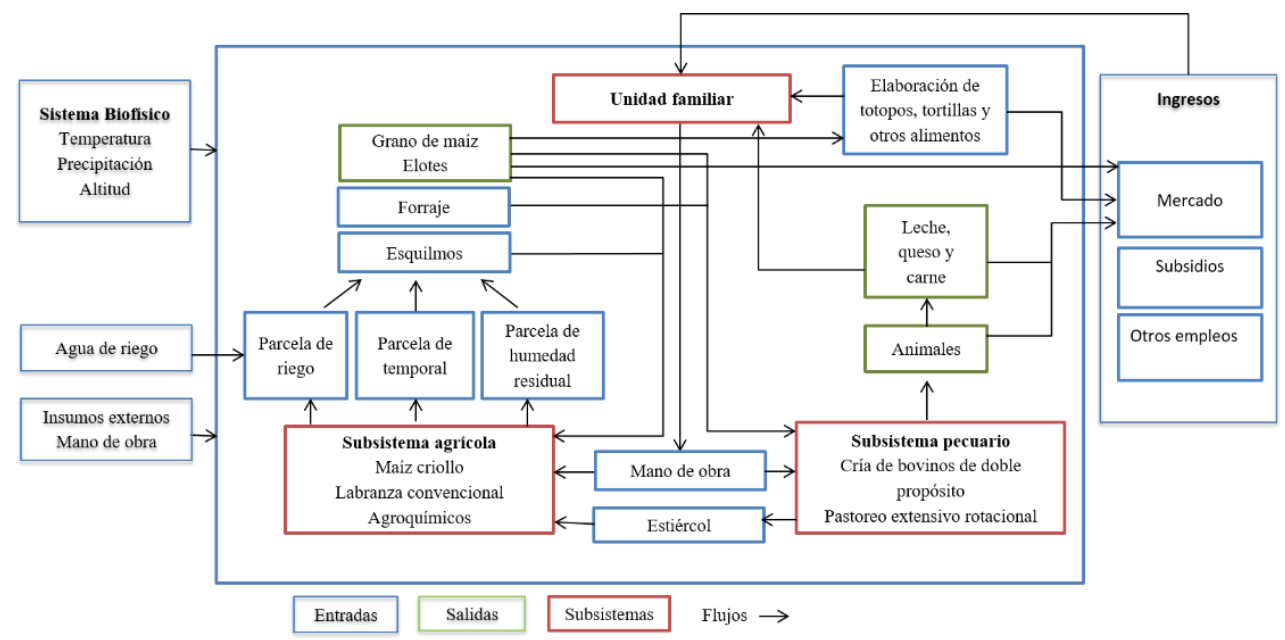

Figura 2. Representación del agroecosistema de maíz en Juchitán de Zaragoza-Chicapa de Castro (AE1).

Según Cervantes-Herrera et al. (2015), la intervención familiar es una forma de capacitación productiva, trasmisión de conocimientos y de valoración de la actividad productiva. Solo $13.6 \%$ de los campesinos pertenece a un grupo de trabajo organizado para la producción de maíz, porcentaje muy bajo que no refleja la participación grupal y no asegura la operatividad y continuidad de los proyectos u otro proceso.

Los campesinos del AE1 siembran maíces criollos de la raza Zapalote Chico, conocido localmente como Xhuba'huini. Es endémico del Istmo de Tehuantepec y de regiones costeras de Oaxaca y Chiapas en altitudes promedio de $600 \mathrm{~m}$, aunque se reportan muestras de otras razas entre los 1 550 y $1900 \mathrm{msnm}$ (Aragón et al., 2005). Las principales características hacen referencia a su precocidad (90 días), resistencia a sequía y plagas.

Es una planta de porte bajo $(110-150 \mathrm{~cm})$ que soporta los fuertes vientos de la zona. Sus mazorcas son cortas con bajo número de hileras de granos semiharinosos, posee el más bajo índice gluma/grano en las razas mexicanas, germen grande y contenido proteico promedio de $12.7 \%$, uno de los más altos de las razas de maíz.

Es base de la alimentación y se utiliza para preparar tortillas, atoles, elote y elaborar totopos (CONABIO, 2010; Cabrera-Toledo et al. 2016). La preferencia de los genotipos locales ocurre en varios estados de México (Herrera et al., 2002; Vásquez-Carrillo et al., 2010); por ejemplo, en Chiapas (Aguilar-Jiménez et al., 2011) y Puebla (Zagoya, 2015), se debe a su menor costo y a las características deseables para la elaboración de alimentos.

El cultivo inicia con la preparación mecanizada del terreno $(90.9 \%)$ y tradicional $(9.1 \%)$ con arado egipcio. La siembra es bajo dos métodos: tradicional con el uso de fuerza animal y humana (68.2\%) y con sembradoras mecánicas $(31.8 \%)$. Por cuestiones de tiempo, dado el ciclo corto del maíz Zapalote Chico, los campesinos combinan tecnología convencional (labranza primaria) y tradicional (labranza secundaria, surcado, siembra y deshierbe), igualmente reportado por Turiján et al. (2012). 
El $81.81 \%$ fertiliza solo con nitrógeno, 46 a $69 \mathrm{~kg} \mathrm{~N}^{-1}$ en la primera labor o al aporque. Este tratamiento lo logran con dos y tres bulto de urea. Es bajo si se considera la fórmula de 92-46-00 recomendada por Cabrera et al. (2018). La principal plaga es la hormiga arriera (Atta spp.) y para su control utilizan Dicloro Difenil Dicloroetanol esparcido en los nidos.

El 59.1\% de los campesinos en el AE1 controla manualmente las malezas. En San Felipe Teotlalzingo, Puebla, se utiliza la maquinaria para labrar el suelo, pero la siembra, el deshierbe y la fertilización es manual empleando pala y azadón o con la yunta (Zagoya, 2015). Destinan en promedio cinco ha para la siembra de maíz, divididas en dos a cuatro parcelas. El $41 \%$ de los campesinos manejan prácticas para conservar la fertilidad del suelo al incorporar residuos de cosecha y estiércol; rotación de cultivos, cultivos asociados con leguminosas, etc.; eficientes en la agricultura tradicional (Cervantes-Herrera et al., 2015).

Solo $13.6 \%$ de los campesinos del AE1 han participado en programas de asistencia técnica mediante la organización a la que pertenecen. Macedo et al. (2003), indican que los agricultores tradicionales del ejido Cofradía de Suchitlán, Colima, reciben apoyos gubernamentales, pero carecen de capacitación y asistencia técnica, lo que denota la falta de asesores técnicos que posibiliten la adaptación continua de estos $\mathrm{AE}$ que enfrentan retos para mejorar su sustentabilidad.

La cosecha es manual a los tres meses; generalmente contratan jornales externos. Gracias a la precocidad del Zapalote Chico las parcelas se siembran tres veces al año bajo tres condiciones de humedad: temporal, riego y humedad residual. Esta última es conocida como siembra de Igudxa, que aprovecha la humedad residual del temporal y los serenos de octubre a diciembre.

Se identificó un sistema de cultivo único bajo temporal y dos sistemas de cultivo múltiple, uno bajo riego y temporal (31.8\%), y el otro bajo riego, temporal y humedad (13.6\%), con una producción acumulada promedio de $3665 \mathrm{~kg} \mathrm{ha}^{-1} \mathrm{año}^{-1}$, baja si se compara con rendimientos promedios de 2 $950 \mathrm{~kg} \mathrm{ha}{ }^{-1}$ ciclo $^{-1}$ (Cabrera et al., 2018). Por ciclo de cultivo se contratan 10 jornales de 30 requeridos para la limpia de canales, siembra, aporque, riego y cosecha.

La principal fuente de ingreso de las UPF es la agricultura, por la venta de maíz y la ganadería (venta de leche, queso y animales en mercados locales). Similar a los AE de Tierra Caliente, Michoacán (Villa-Méndez et al., 2008) y el Ejido Cofradía Suchitlán, Colima (Macedo et al., 2003), donde 60 y $83 \%$ de los campesinos, respectivamente, dependen económicamente de la UPC.

El ingreso promedio mensual por UPC es de $\$ 6750.00$ y sobrepasa lo reportado por Serrano-Ojeda et al. (2016) para la región centro de Puebla, con un ingreso en los de mayor seguridad alimentaria de \$2 566.00 mensuales. En el AE1, 27\% complementa ingresos con el apoyo del PROAGRO Productivo, útil para el proceso productivo y otras necesidades básicas. Las UPC en la región que son apoyadas es menor a las de Tulancingo, Hidalgo, donde 36\% de los maiceros lo reciben, posiblemente a que existen organizaciones más consolidadas (Ayala-Garay et al., 2013).

El destino de la producción varía, 36.4\% de las UPC del AE1 el maíz para el autoconsumo, 45.4\% comercializa solo excedentes y $18.2 \%$ restante vende todo lo producido debido a las necesidades de ingreso o a la falta de infraestructura para el almacenamiento. En Tlaxcala, 40\% usa el maíz 
para el autoconsumo, $2 \%$ para la venta y 58\% para ambos (Damián et al., 2007b; Damián et al., 2008). Esto difiere con lo reportado en el ejido Cofradía Suchitlán, Colima, donde $83 \%$ de las UPC satisface sus necesidades de alimentación (Macedo et al., 2003).

Esto indica que el maíz atiende distintas necesidades al venderse directamente o comercializarse trasformado debido a sus diversas formas de consumo. La comercialización es local y se vende por litro (750 g), a un precio de $\$ 7.00$. Económicamente el alquiler de la mano de obra es importante en este AE, al ser jornaleros fuera de la UPC con un ingreso de $\$ 170.00$ jornal $^{-1} \mathrm{y}$ un promedio de 8 jornales ha- ${ }^{-1}$ por ciclo, aunque puede ser de hasta 12 jornales ha ${ }^{-1}$ por ciclo de maíz.

\section{Agroecosistema de maíz en Álvaro Obregón-Emiliano Zapata (AE2)}

El AE2 se ubica a una altitud de $6 \mathrm{~m}$. La temperatura máxima es de $29.8^{\circ} \mathrm{C}$, la media anual de $26{ }^{\circ} \mathrm{C}$ y la precipitación promedio de $942 \mathrm{~mm}$ anuales. Estructuralmente, el AE2 presenta una variante en el subsistema pecuario con la crianza de ovinos bajo un régimen extensivo de pastoreo, en contraste con los AE1 y 3 que se dedican a la crianza de bovinos de doble propósito (cría y leche).

Los campesinos son zapotecos (95\%) y mestizos (5\%) (No hablantes del zapoteco) y no pertenecen a ninguna organización para la producción de maíz. Las UPF se integran por 6.5 personas en promedio. El jefe de familia es el encargado de la UPC y colaboran en ella de 2 a 3 de los hijos.

Serrano-Ojeda et al. (2016) reportan que en el centro de Puebla las UF se integran por cuatro personas y dos colaboran activamente en la UP. De acuerdo con la SAGARPA (2017), la agricultura tradicional se basa en el uso intensivo de la mano de obra familiar.

De igual manera, cultivan el maíz Zapalote Chico. Para reducir tiempos, la preparación del terreno es mecanizado (100\%) y la siembra es mecánica (75\%) y tradicional con arado egipcio (25\%). El $80 \%$ emplea fertilizantes nitrogenados $\left(46 \mathrm{~kg} \mathrm{~N} \mathrm{ha}^{-1}\right)$, al momento del aporque y mateado, lejos de las dosis de 92-46-00 ( $\mathrm{kg} \mathrm{ha}^{-1}$ de nitrógeno, fósforo y potasio, respectivamente) recomendada por Cabrera et al. (2018). No utilizan insecticidas, ya que las plagas no son significativas.

El control de arvenses es manual y solo se realiza en los bordes de las parcelas (90\%). El 50\% incorpora cantidades diversas de residuos de cosecha y estiércol del subsistema pecuario anterior a la preparación del suelo para conservar la fertilidad (Figura 3).

Los agricultores de la subcuenca del Río Atoyac, Oaxaca, indican que es necesario fertilizar, realizar dos deshierbes, rotar con leguminosas e incorporar estiércol y residuos para lograr cosechas (Ruiz-Vega y Silva-Rivera, 2006). Además, son fuente importante de nitrógeno orgánico (SalazarSosa et al., 2009; Salazar-Sosa et al., 2010), permiten el desarrollo de microorganismos que promueven la fertilidad (Damián et al., 2013), mejora estructura y textura, aumenta la aireación, penetración de raíces, retiene agua y se logran plantas fuertes y sanas resistentes a plagas y enfermedades (Aguilar et al., 2003; Damián et al., 2008). 


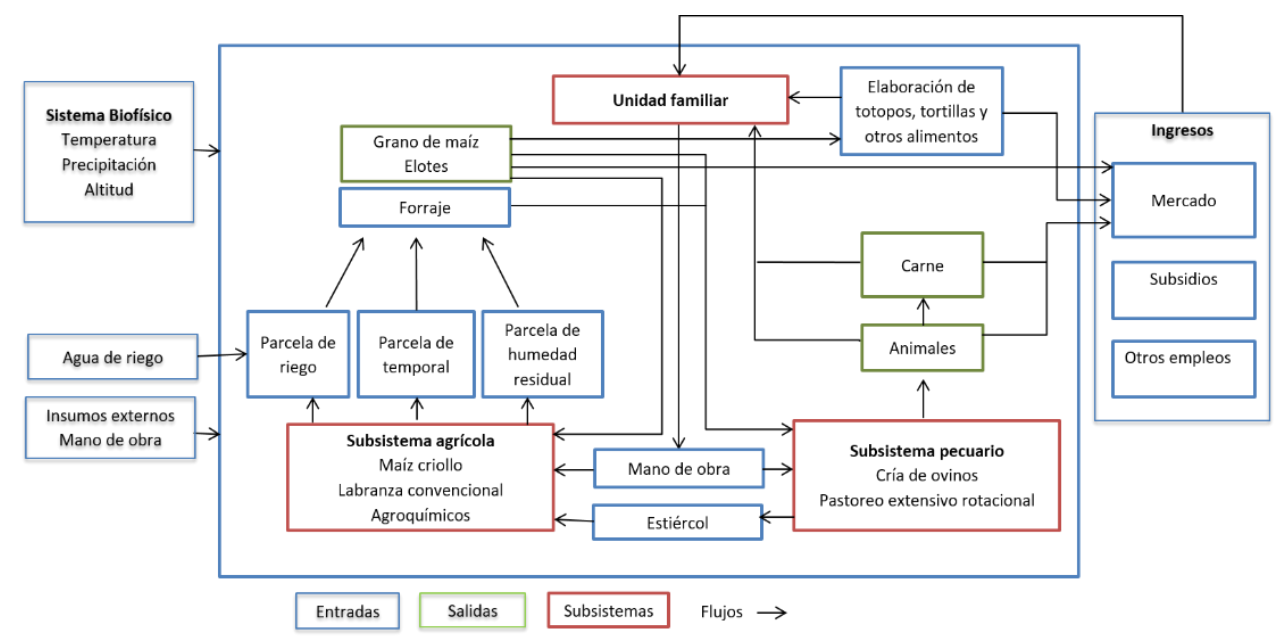

Figura 3. Representación del agroecosistema de maíz en Álvaro Obregón-Emiliano Zapata (AE2).

Este esquema es similar al de Tlaxcala, donde $84.4 \%$ usa fertilizantes nitrogenados y $66.4 \%$ incorporan estiércol al suelo (Damián et al., 2007a; Damián et al., 2007b). Igualmente, agricultores mayas de Petén, Guatemala, no utilizan fertilizantes ni abonos para la milpa y el control de malezas es manual (Lara et al., 2012). En el AE2, cada productor cultiva 3.6 ha en 1 y 2 parcelas.

De acuerdo con Ayala-Garay et al. (2013), es similar a Tulancingo, Hidalgo; donde cultivan 3.36 ha en promedio, pero menor a Tlaxcala (Damián et al., 2008), donde son 5 ha y mayor con respecto a la región centro de Puebla con 2.36 ha en promedio (Serrano-Ojeda et al., 2016).

La superficie de cultivo es importante ya que, a mayor superficie, mayor producción, lo que posibilita la autosuficiencia y excedentes para la venta, siempre que haya los insumos necesarios (Pat-Fernández et al., 2011). En este AE también se realizan tres cultivos al año: sistema simple de temporal (75\% de los agricultores), sistemas múltiples de riego y temporal (75\%) y sistema de riego, temporal y humedad (10\%); con lo que se genera una producción acumulada de 3312 $\mathrm{kg} \mathrm{ha}^{-1} \mathrm{año}^{-1}$.

En este no existe la asesoría técnica para el cultivo del maíz. En cada ciclo del cultivo se contratan en promedio cinco jornales para apoyar las actividades de siembra, aporque y cosecha. Las UPF dependen de las actividades agrícolas, venta directa de maíz y elaboración de totopo, actividades ganaderas (venta de animales) y del trabajo asalariado de los hijos fuera de la UPC, con un promedio de 12 jornales por ciclo por cada uno de los integrantes.

En el ingreso se contempla el apoyo del PROAGRO Productivo (85\% de los productores); para un ingreso promedio mensual de $\$ 3675.00$. Esta diversidad ocurre en todo el país y es similar a la del Valle de Tehuacán, Puebla, donde los campesinos cultivan, crían cabras, comercializan leña y especies no maderables (Aguilar et al., 2003). 
El maíz es para el autoabasto (80\%) y venta (20\%), similar a 96\% identificado en Tulancingo, Hidalgo (Ayala-Garay et al., 2013). Al igual que el AE1, la venta es local y el litro de maíz se vende a $\$ 7.00$. El pago por la mano de obra es de $\$ 130.00$ por jornal.

\section{Agroecosistema de maíz en La Venta-La Ventosa (AE3)}

El AE3 se ubica en altitudes de $39 \mathrm{~m}$, con temperaturas máximas de $32.8^{\circ} \mathrm{C}$ y la media anual de $27.5^{\circ} \mathrm{C}$, con precipitaciones anuales de $1007 \mathrm{~mm}$, superiores al rango óptimo. Los campesinos son zapotecas y mestizos $(50 \%)$.

Las UPF son de seis individuos y las actividades están a cargo del jefe de familia y participan ocasionalmente dos de los hijos. Distinto a los cuatro en la región centro de Puebla (Serrano-Ojeda et al., 2016). Asimismo, los agricultores no pertenecen a grupos de trabajo u organización local, situación que no permite generar economías de escala (Ayala-Garay et al., 2013).

Estructuralmente, el AE3 se diferencia de los AE2 y AE1 en el subsistema agrícola, al sembrarse en estos últimos bajo condiciones de humedad, aunque el subsistema pecuario resulta similar con el AE1, al contar con ganadería de doble propósito (Figura 4).

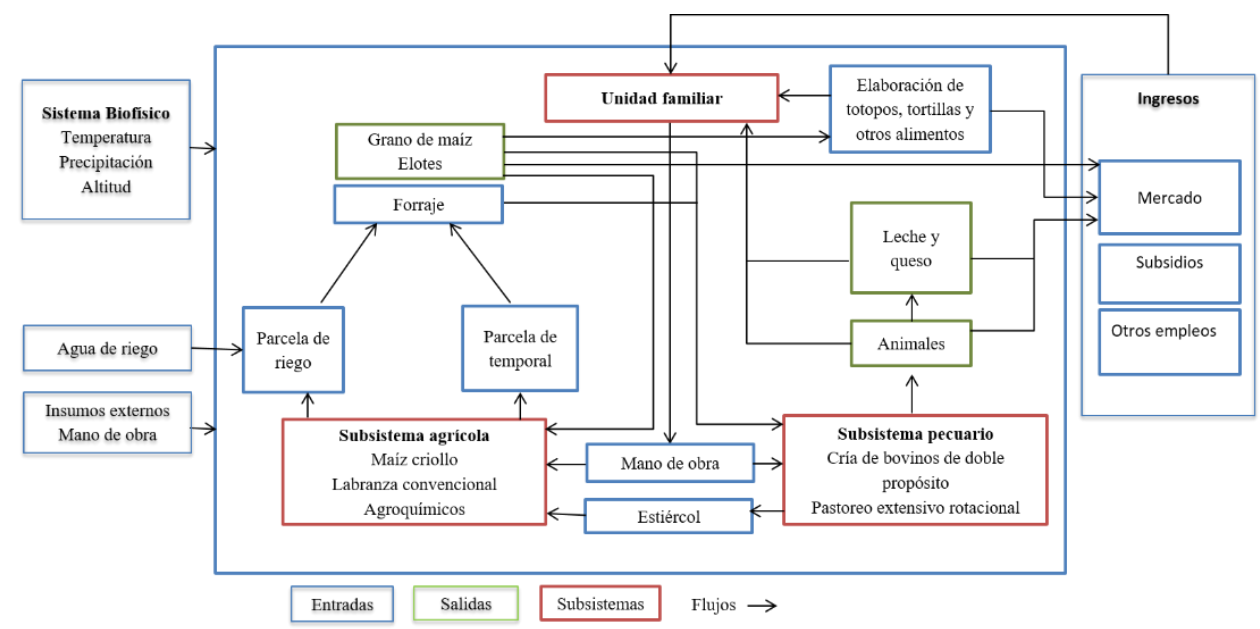

Figura 4. Representación del agroecosistema de maíz en La Venta-La Ventosa (AE3).

La semilla es de Zapalote Chico seleccionada de la cosecha anterior debido a características de adaptación a la región y para la elaboración de tortillas y totopos para el autoconsumo (AyalaGaray et al., 2013). La preparación del terreno es mecanizada (100\%) y la siembra es mecánica (94.4\%). El 83.3\% emplea fertilizantes nitrogenados a razón de 46 a $69 \mathrm{~kg} \mathrm{~N} \mathrm{ha}^{-1}$, a base de urea. Se aplica mateado un día antes del aporque. No se presentarse plagas de consideración. Tradicionalmente incorporan residuos de cosecha y estiércol (77.8\%). En promedio siembras 4.7 ha distribuidas en 1.2 parcelas, esto es, superficies más compactas que en los otros AE.

Esta superficie es superior a la utilizada en Hidalgo, donde se cultivan 2.5 ha de maíz en promedio (Ayala-Garay et al., 2010). Solo 27.7\% de los productores recibe ocasionalmente asesoría técnica. Esta situación se presenta en varios estados del país. En Tlaxcala, 91.6\% de los productores de maíz carecen de asistencia técnica (Damián et al., 2007a; Damián et al., 2007b). 
Este factor es de suma importancia, ya que refuerza las habilidades y capacidades de los agricultores y es decisivo para incrementar la producción agrícola (Damián et al., 2008; AyalaGaray et al., 2013). En el AE3 se detectaron tres sistemas de cultivo con base a la humedad, riego (61\% de los productores), temporal (22\%) y un sistema múltiple bajo riego y temporal (16.6\%).

En este último es posible obtener una producción acumulada de $3244 \mathrm{~kg} \mathrm{ha}^{-1}$ año $^{-1}$. En el Valle de Tehuacán, Puebla, los campesinos tienen agricultura de riego y temporal para producir mayor alimento al año (Aguilar et al., 2003). En este AE, para cada ciclo de cultivo contratan en promedio ocho jornales para actividades de limpia de canales, siembra, aporque, riego y cosecha.

El ingreso promedio mensual de las UPF de este AE es \$6 000.00, por actividades ganaderas y agrícolas. Al igual que los AE1 y AE2, 94.4\% de los productores cuenta con el apoyo del PROAGRO Productivo. El maíz es para el autoabasto $(88.9 \%)$ y para la venta (11.1\%). Cifras similares a las de Cuetzalan, Puebla, donde $18 \%$ se comercializa (Damián et al., 2013).

El grano se vende localmente por litro a $\$ 8.00$. La mano de obra contratada genera un gasto de $\$ 150.00$ jornal $^{-1}$. Los integrantes de este $\mathrm{AE}$ también se desempeñan como jornaleros con 12 jornales ciclo $^{-1}$ para complementar el ingreso.

\section{Conclusiones}

Se identificaron tres AE de maíz: AE1 (Zaragoza-Chicapa de Castro); AE2 (Álvaro ObregónEmiliano Zapata) y AE3 (La Venta-La Ventosa), diferentes en aspectos biofísicos, económicos y sociales, aunque muy similares en lo técnico. Relevantes al permitir el sustento de las UPF a pesar de que se involucran de forma ocasional de dos a tres personas en las actividades agrícolas. Ambientalmente los AE son parecidos y se ubican en una gradiente altitudinal entre 6 y $39 \mathrm{~m}$, con una temperatura media anual de 26 y $27.5^{\circ} \mathrm{C}$ y una precipitación anual de 943 y $1007 \mathrm{~mm}$.

La precocidad del Zapalote Chico y el riego permiten tres cosechas al año bajo sistemas de cultivos únicos (temporal o riego) y sistemas múltiples (riego y temporal, riego, temporal y humedad y temporal y humedad). Étnicamente existe persistencia de la cultura zapoteca en los tres $\mathrm{AE}$, aunque difieren los AE1 y 2 del AE3, al poseer este último por población zapoteca $(50 \%)$ y mestiza $(50 \%)$.

Socioeconómicamente los tres AE son importantes al incluir de dos a tres personas por UPF en las actividades productivas y generan de 5 a 10 jornales ha $^{-1}$ ciclo $^{-1}$ y 12 jornales ha $^{-1}$ ciclo $^{-1}$ fuera de las UPC, lo que mejora el ingreso y el apoyo social; lo que subsana en parte, la baja capacidad organizativa y se manifiesta en el AE1 con $13.6 \%$ de productores organizados y que repercute en la baja capacidad para recibir acompañamiento técnico (13.6, 0 y $27.7 \%$ para los AE1, 2 y 3 , respectivamente).

Los AE1 y 3 tienen los mayores ingresos $\$ 6750.00$ y $\$ 6000.00$, respectivamente; superiores al AE2 (\$3 675.00 mensuales), debido a la baja superficie (3.6 ha), bajo precio de los jornales (\$130.00), baja cantidad de jornales contratados (5), bajos excedentes vendidos (20\%) y al sistema de ovinos en pastoreo intensivo, lo que limita el ingreso al no vender leche, queso y carne. 


\section{Literatura citada}

Aguilar, J.; Illsley, C. y Marielle, C. 2003. El maíz como cultivo. In: Esteva, G. y Marielle, C. (Cords.). Sin maíz no hay país. Consejo Nacional de la Cultura (CONACULTA). México, DF. Cap. II. 83-122 pp.

Aguilar-Jiménez, C. E.; Tolón-Becerra, A. y Lastra-Bravo, X. 2011. Evaluación integrada de la sostenibilidad ambiental, económica y social del cultivo de maíz en Chiapas, México. Revista de la Facultad de Ciencias Agrarias. Universidad Nacional de Cuyo. 43(1):155174. https://doi.org/10.21041/conpat2019/v2pat36.

Aragón-Cuevas, F.; Taba, S.; Castro-García, F. H.; Hernández-Casillas, J. M.; Cabrera-Toledo, J. M.; Alcalá, L. O. and Ramírez, N. D. 2005. In situ conservation and use of local maize races in Oaxaca, Mexico: A participatory and decentralized approach. In: Taba, S. (Ed.). Latin American maize germplasm conservation: regeneration, in situ conservation, core subsets, and prebreeding; proceedings of a workshop held at CIMMYT. April 2003. México, DF. 26-38 pp.

Ayala-Garay, A. V.; Sangerman-Jarquín, D. M.; Schwentesius, R. R.; Damián, H. M. Á. y Juárez, R. C. G. 2010. Fortalecimiento de la competitividad del sector agropecuario en Hidalgo. Rev. Mex. Cienc. Agríc. 1(2):233-245. https://doi.org/10.29312/remexca.v2i4.1636.

Ayala-Garay, A. V.; Schwentesius-Rindermann, R.; Preciado-Rangel, P.; Almaguer-Vargas, G. y Rivas-Valencia, P. 2013. Análisis de rentabilidad de la producción de maíz en la región de Tulancingo, Hidalgo, México. Agric. Soc. Des. 10(4):381-395. https://doi.org/ 10.22231/ asyd.v10i4.132.

Cabrera, T. J. M.; Carballo, C. A. y Aragón, C. F. 2018. Evaluación agronómica de maíces raza Zapalote Chico en la región istmeña de Oaxaca. Rev. Mex. Cienc. Agríc. Pub. Esp. No. 11. 2075-2082. https://doi.org/10.29312/remexca.v0i11.775.

Cabrera-Toledo, J. M.; Carballo-Carballo, A.; Mejía-Contreras, J. A.; García-de los Santos, G. y Vaquera-Huerta, H. 2016. Calidad de grano de los maíces criollos sobresalientes de la raza Zapalote Chico. Pérez, F.; Figueroa, E. y Godínez, L. (Eds.). Producción, comercialización y medio ambiente. Handbook T-I. ${ }^{\circ}$ ECORFAN. Texcoco de Mora, México. 63-72 pp.

Cervantes-Herrera, J.; Castellanos, J. A.; Pérez-Fernández, Y. y Cruz, L. A. 2015. Tecnologías tradicionales en la agricultura y persistencia campesina en México. Rev. Mex. Cienc. Agríc. 2:381-389.

Colmenares, E. A. M. 2012. Investigación-acción participativa: una metodología integradora del conocimiento y la acción. Universidad Pedagógica Experimental Libertador. Voces y Silencios: Rev. Latinoam. Ed. 3(1):102-115. pp

CONABIO. 2010. Comisión Nacional para el Conocimiento y Uso de la Biodiversidad. Argumentación para conservar las razas de maíces nativos de México. México, DF. http://www.biodiversidad.gob.mx/genes/pdf/proyecto/Anexo6_ReunionesTalleres/Tabla\% 20razas_marzo\%202010.pdf.

Damián, H. M. Á.; Cruz, L. A.; Ramírez, V. B.; Romero, A. O.; Moreno, L. S. y Reyes, M. L. 2013. Maíz, alimentación y productividad: modelo tecnológico para productores de temporal de México. Agric. Soc. Des. 10(2):157-176.

Damián, H. M. Á.; López, O. J. F.; Ramírez, V. B.; Parra, I. F.; Paredes, S. J. A.; Gil, M. A. y Cruz, L. A. 2008. Hombres y mujeres en la producción de maíz: un estudio comparativo en Tlaxcala. Región y Sociedad. 20(42):63-94. https://doi.org/10.22198/ rys.2008.42.a509. 
Damián, H. M. Á.; Ramírez, V. B.; Parra, I. F.; Paredes, S. J. A.; Gil, M. A.; Cruz, L. A. y López, O. J. F. 2007a. Apropiación de tecnología por productores de maíz en el estado de Tlaxcala, México. Agric. Téc. Méx. 33(2):163-173.

Damián, H. M. Á.; Ramírez, V. B.; Parra, I. F.; Paredes, S. J. A.; Gil, M. A.; López, O. J. F. y Cruz, L. A. 2007b. Tecnología agrícola y territorio: el caso de los productores de maíz de Tlaxcala, México. Investigaciones Geográficas. 63:35-55.

González, C. M. E.; Palacios, R. N.; Espinoza, B. A. y Bedoya, S. C. A. 2013. Diversidad genética en maíces nativos mexicanos tropicales. Rev. Fitotec. Mex. 36(6):239-338. https://doi.org/10.35196/rfm.2013.3-s3-a.329.

Hernández Xolocotzi, E. y Ramos R. A. 1977. Metodología para el estudio de agroecosistemas con persistencia de tecnología agrícola tradicional. In: Hernández-Xolocotzi, E. 1985 (Ed.). Xolocotzia. Rev. Geogr. Agríc. Universidad Autónoma Chapingo (UACH), Texcoco, Estado de México. Tomo 1. 189-194 pp.

Herrera, C. B. E.; Macías-López, A.; Díaz, R. R.; Valadez, R. M. y Delgado, A. A. 2002. Uso de semilla criolla y caracteres de mazorca para la selección de semilla de maíz en México. Rev. Fitotec. Mex. 25(1):17-23.

INEGI. 2010a. Instituto Nacional de Estadística y Geografía. Anuario estadístico de Oaxaca. http://www.inegi.org.mx/est/contenidos/espanol/sistemas/aee10/info/oax/mapas.pdf.

INEGI. 2010b. Instituto Nacional de Estadística y Geografía. Compendio de información geográfica municipal 2010. Heroica Ciudad de Juchitán de Zaragoza. 9 p.

INEGI. 2014. Instituto Nacional de Estadística y Geografía. Anuario estadístico y geográfico de Oaxaca. México. 1444 p.

Lara, P. E.; Caso, B. L. y Aliphat, F. M. 2012. El sistema milpa roza, tumba y quema de los mayas Itzá en San Andrés y San José, Petén, Guatemala. Ra Ximhai. 8(2):69-90. https://doi.org/10.35197/rx.08.02.e.2012.06.el.

López, R. G.; Santacruz, V. A.; Muñoz, O. A.; Castillo, G. F.; Córdova, T. L. y Vaquera, H. H. 2009. Perfil isoenzimático de maíces nativos del Istmo de Tehuantepec, Oaxaca, México. II. Variación dentro de grupos. Rev. Fitotec. Mex. 32(3):177-188.

López, R. G.; Santacruz, V. A.; Muñoz, O. A.; Castillo, G. F.; Córdova, T. L. y Vaquera, H. H. 2010. Perfil isoenzimático de maíces nativos del Istmo de Tehuantepec, Oaxaca, México. I. Caracterización de grupos. Rev. Fitotec. Mex. 33(1):1-10.

López-Romero, G.; Santacruz-Varela, A.; Muñoz-Orozco, A.; Castillo-González, F.; CórdovaTéllez, L. y Vaquera-Huerta, H. 2005. Caracterización morfológica de poblaciones nativas de maíz del Istmo de Tehuantepec, México. Rev. Interciencia. 30(5):284-290.

Macedo, R.; Galina, M. A.; Zorrilla, J. M.; Palma, J. M. y Pérez-Guerrero, J. 2003. Análisis de un sistema de producción tradicional en Colima, México. Archivos de Zootecnia. 52(200):463-474.

Masera, O.; Astier, M. y López-Ridaura, O. 2000 Sustentabilidad y manejo de recursos naturales. El marco de evaluación MESMIS. Grupo Interdisciplinario de Tecnología Rural Apropiada (GIRA A. C.). Editorial Mundiprensa. México, DF. 103 p.

Pat-Fernández, L. A.; Nahed-Toral, J.; Parra-Vázquez, M. R.; García-Barrios, L.; NazarBeutelspacher, A. y Bello-Baltazar, E. 2011. Influencia de las estrategias de ingresos y las políticas públicas sobre la seguridad alimentaria en comunidades rurales mayas del norte de Campeche, México. Trop. Subtrop. Agroecosys. 14(1):77-89.

Ruiz-Vega, J. y Silva-Rivera, M. E. 2006. Caracterización de dos agroecosistemas presentes en suelos de ladera de la subcuenca Sur Oeste del Río Atoyac, Oaxaca. Naturaleza y Desarrollo. 4(2):5-12. 
Salazar-Sosa, E.; Trejo-Escareño, H. I.; López-Martínez, J. D.; Vázquez-Vázquez, C.; SerratoCorona, J. S.; Orona-Castillo, I. y Flores-Márgez, J. P. 2010. Efecto residual de estiércol bovino sobre el rendimiento de maíz forrajero y propiedades del suelo. Terra Latinoam. 28(4):381-390.

Salazar-Sosa, E.; Trejo-Escareño, H. I.; Vázquez-Vázquez, C.; López-Martínez, J. D.; FortisHernández, M.; Zúñiga-Tarango, R. y Amado-Álvarez, J. P. 2009. Distribución de nitrógeno disponible en suelo abonado con estiércol bovino en maíz forrajero. Terra Latinoam. 27(4):373-382.

Salinas, M. Y.; Aragón, C. F.; Ybarra, M. C.; Aguilar, V. J.; Altunar, L. B. y Sosa. M. E. 2013. Caracterización física y composición química de razas de maíz de grano azul/morado de las regiones tropicales y subtropicales de Oaxaca. Rev. Fitotec. Mex. 36(1):23-31. https://doi.org/10.35196/rfm.2013.1.23.

SAGARPA. 2017. Secretaría de Agricultura, Ganadería, Desarrollo Rural, Pesca y Alimentación. Agricultura de autoconsumo. http://www.sagarpa.gob.mx/Delegaciones/ oaxaca/Paginas/Autoconsumo2013.aspx.

Selener, D.; Endara, N. y Carvajal, J. 1999. Sondeo rural participativo: guía práctica. Instituto internacional de reconstrucción rural (IIRR). Segunda edición. Quito, Ecuador. 131 p.

Serrano-Ojeda, M. L.; Calderón-Sánchez, F.; Vargas-López, S.; López-Sánchez, H.; AntonioLópez, P.; Martínez-Trejo, G.; Cortés-Díaz, E. y Salcido-Ramos, B. A. 2016. Características estructurales y productivas de hogares con diferente grado de seguridad alimentaria en Puebla. Agric. Soc. Des. 13(4):547-563. https://doi.org/10.22231/ asyd.v13i4.493.

SIAP. 2015. Servicio de Información Agroalimentaria y Pesquera. Anuario estadístico de la producción. http://infosiap.siap.gob.mx/aagricola_siap_gb/ientidad/index.jsp.

SIAP. 2016. Servicio de Información Agroalimentaria y Pesquera. Atlas agroalimentario México D.F. pp. 102-105.

SIAP. 2017. Servicio de Información Agroalimentaria y Pesquera. Anuario estadístico de la producción agrícola. http://infosiap.siap.gob.mx/aagricola_siap_gb/icultivo/index.jsp.

Turiján, A. T.; Damián, H. M. A.; Ramírez, V. B.; Juárez, S. J. P. y Estrella, C. N. 2012. Manejo tradicional e innovación tecnológica en cultivo de maíz en San José Chiapa, Puebla. Rev. Mex. Cienc. Agríc. 6(3):1085-1100. https://doi.org/10.29312/remexca.v3i6.1361.

UABJO. 2014. Universidad Autónoma Benito Juárez de Oaxaca. Plan municipal de desarrollo de la Heroica Ciudad de Juchitán de Zaragoza 2014-2016.

Vázquez-Carrillo, M.; Pérez, C. J. P.; Hernández, C. J. M.; Marrufo, D. M. y Martínez, R. E. 2010. Calidad de grano y de tortillas de maíces criollos del altiplano y Valle del Mezquital, México. Rev. Fitotec. Mex. 33(4):49-56.

Vilaboa-Arroniz, J.; Díaz-Rivera, P.; Ruiz-Rosado, O.; Platas-Rosado, D. E.; González-Muñoz, S. y Juárez-Lagunes, F. 2009. Caracterización socioeconómica y tecnológica de los agroecosistemas con bovinos de doble propósito de la región del Papaloapan, Veracruz, México. Trop. Subtrop. Agroecosys. 10(1):53-62.

Villa-Méndez, C. I.; Tena, M. J.; Tzintzun, R. y Val, D. 2008. Caracterización de los sistemas ganaderos en dos comunidades del municipio de Tuzantla de la región de Tierra Caliente, Michoacán. Av. Investig. Agrop. 12(2):45-57.

Zagoya, M. J. 2015. Sistema tradicional utilizado en la producción de maíz en La Sierra Nevada de Puebla, México. Rev. Caribeña Cienc. Soc. 01(08):1-6. 